\title{
Clinical utility of arterial spin labeling imaging in disorders of the nervous system
}

\author{
Sauson Soldozy, BA, Jacob Galindo, BS, Harrison Snyder, BS, Yusuf Ali, BS, Pedro Norat, MD, \\ Kaan Yağmurlu, MD, Jennifer D. Sokolowski, MD, PhD, Khadijeh Sharifi, PhD, Petr Tvrdik, PhD, \\ Min S. Park, MD, and M. Yashar S. Kalani, MD, PhD
}

Department of Neurological Surgery, University of Virginia Health System, Charlottesville, Virginia

\begin{abstract}
Neuroimaging is an indispensable tool in the workup and management of patients with neurological disorders. Arterial spin labeling (ASL) is an imaging modality that permits the examination of blood flow and perfusion without the need for contrast injection. Noninvasive in nature, ASL provides a feasible alternative to existing vascular imaging techniques, including angiography and perfusion imaging. While promising, ASL has yet to be fully incorporated into the diagnosis and management of neurological disorders. This article presents a review of the most recent literature on ASL, with a special focus on its use in moyamoya disease, brain neoplasms, seizures, and migraines and a commentary on recent advances in ASL that make the imaging technique more attractive as a clinically useful tool.
\end{abstract}

https://thejns.org/doi/abs/10.3171/2019.9.FOCUS19567

KEYWORDS arterial spin labeling; functional MRI; neuroradiology; cerebrovascular; neurooncology

$\Lambda$ RTERIAL spin labeling (ASL) is a neuroimaging technique that noninvasively quantifies cerebral blood flow (CBF). Introduced in the early 1990s, ASL provides a superior alternative to blood oxygen leveldependent (BOLD) imaging in determining cerebral perfusion; notably, BOLD imaging suffers from poor spatial resolution and questionable neuronal specificity given the venous contrast drainage. ${ }^{3}$

Other techniques that measure brain perfusion include CT perfusion imaging, dynamic susceptibility contrastperfusion weighted imaging (DSC-PWI), positron emission tomography (PET), and single-photon emission CT (SPECT). ${ }^{12,14}$ The value of ASL, a functional MRI (fMRI) sequence, lies in its utilization of endogenous blood-based water molecules as a tracer along with its lack of ionizing radiation..$^{15}$ This omits the need for exogenous tracer or contrast injection and excludes unnecessary radiation exposure to patients. Serving as a physiological correlate to adenosine triphosphate (ATP) consumption, CBF is a clinically useful parameter that measures neuronal activity and has the ability to map differential perfusion and energy utilization in different locations of the brain. 2,12,20,30 For this reason, by using CBF as a surrogate, ASL pro- vides a promising and safe approach to measuring perfusion patterns and overall neuronal activity. ${ }^{2}$ Given that the brain has a persistent state of intrinsic metabolic activity in the resting state, ASL provides the opportunity to detect and monitor alterations in tissue perfusion indicative of brain injury and dysfunction.

These include neurovascular disorders such as stroke, vasospasm, moyamoya disease (MMD), and Sturge-Weber syndrome, which are disease processes characterized by decreased CBF. Vascular shunts (dural arteriovenous fistula, arteriovenous malformation) and tumors typically reflect increased CBF on ASL imaging. Mixed CBF is indicative of other neurological disorders including seizure, migraine, and posterior reversible encephalopathy syndrome. ${ }^{38}$ With these differences in CBF based on pathology now elucidated, ASL can help to delineate otherwise difficult to discern lesions on brain imaging and aid in diagnosing and managing neurological disorders. In the recent decade, ASL has become more refined and more easily implemented. Thus, there has been increased interest in exploring the clinical application of ASL, especially as technological barriers to improved signal detection are being removed.

ABBREVIATIONS ASL = arterial spin labeling; CASL = continuous ASL; CBF = cerebral blood flow; DSC-PWI = dynamic susceptibility contrast-perfusion weighted imaging; ECA = external carotid artery; EEG = electroencephalography; ICA = internal carotid artery; MMD = moyamoya disease; $\mathrm{mTI}-\mathrm{ASL}=$ multi-inversion time ASL; PASL = pulsed ASL; pCASL = pseudo-continuous ASL; PET = positron emission tomography; PLD = postlabeling delay; $\mathrm{RF}$ = radiofrequency; ROI = region of interest; $\mathrm{SNR}=$ signal-to-noise ratio; STA-MCA = superficial temporal artery-middle cerebral artery; T-ASL = territorial ASL.

SUBMITTED August 2, 2019. ACCEPTED September 16, 2019.

INCLUDE WHEN CITING DOI: 10.3171/2019.9.FOCUS19567. 
In this review, we provide a background of ASL, focusing on recent developments with a commentary on its mechanism and various sequencing options. Additionally, a discussion of ASL and its utility in the setting of neurovascular, neoplastic, neurodegenerative, and other diseases of the nervous system is presented.

\section{Overview of ASL}

Although the process was initially met with skepticism, Detre et al. provided the first description of perfusion imaging to measure changes in CBF in rat brains. ${ }^{6,20}$ This same author group obtained perfusion images of freezeinjured rat brain in another study, providing early evidence of the ability to detect regional abnormalities in perfusion with this imaging technique..$^{41}$ The greater part of the 21st century has been characterized by improving image acquisition and resolution as high-field scanners continue to be optimized. Dr. Alan P. Koretsky, one of the founding fathers of ASL, believes that as ASL continues to be able to detect relatively small changes in perfusion, its application within the realm of neurological and psychiatric disorders will increase. ${ }^{6}$

\section{Imaging Acquisition}

Acting as a diffusible tracer, the water proton nuclear spins in arterial blood are labeled by the application of a radiofrequency $(\mathrm{RF})$ pulse, causing them to invert at the level of the carotid arteries below the bifurcation.,12,21,30 A postlabeling delay (PLD) is applied to allow the labeled blood to arrive at the region of interest (ROI), where an image is captured via MRI. ${ }^{1,14,21}$ Control images of the area of interest without radiolabeled water are then captured, and these images are then subtracted from the ASL images, thus generating a perfusion image. CBF is then quantified and reported in physiological units of $\mathrm{ml} / 100$ $\mathrm{g} / \mathrm{min} .{ }^{6,26,28}$

RF pulses can be applied in one of several methods: pulsed ASL (PASL), continuous ASL (CASL), and pseudocontinuous ASL (pCASL). In PASL, one short RF pulse is applied to a thick $(15-20 \mathrm{~cm})$ "slab" area of the neck for a duration of 10-20 msec to convert the arterial water spins; this approach is technically easier to implement but carries the disadvantages of a lower signal-to-noise ratio (SNR), lower delivery of labeled magnetization, and greater amount of T1 decay. ${ }^{1,2,6} \mathrm{CASL}$, on the other hand, involves continuous application of the RF pulse (typically approximately 2 seconds) at a labeling plane proximal to the area of interest. This method is complicated by requiring long pulses that cause signal loss due to magnetization transfer effects, as well as by its straining of most commercially available MRI scanners such that most software packages do not include it, making clinical use limited., ${ }^{2,12}$ pCASL is an intermediate technique that involves the rapid application of approximately $1000 \mathrm{RF}$ pulses to the labeling plane at a goal rate of about $1 / \mathrm{msec}^{1}$ This technique helps to increase the SNR and decrease T1 decay as compared to those with PASL, but it also decreases the magnetization transfer effects seen in CASL and is more compatible with the hardware capabilities of most MRI scanners. ${ }^{1,2,6,14}$
In 2014, a consensus statement was released by the International Society for Magnetic Resonance in Medicine (ISMRM) and the European consortium ASL in Dementia (AID), which details the current recommendations for implementation of ASL for perfusion imaging in the clinical setting. ${ }^{1}$ These recommendations include the use of a 3-T MRI scanner, a pCASL pulse sequence for labeling with RF pulse spacing as short as possible, stratification of labeling duration by age, and a segmented 3D multi-echo readout.

\section{Moyamoya Disease}

MMD is characterized by idiopathic progressive supraclinoid internal carotid artery (ICA) stenosis along with abnormal skull base vasculature. Symptoms include transient ischemic attacks in both pediatric and adult populations, while intracranial hemorrhages are more common in adults. Diagnosis is performed via catheter angiography, with high-resolution MRI used to determine the extent of vessel wall disease and narrowing. ${ }^{8}$ The mainstay treatment for MMD is extracranial-intracranial bypass surgery, with superficial temporal artery-middle cerebral artery (STA-MCA) bypass shown to improve patient outcomes and prevent hemodynamic stress and rebleeding. ${ }^{36}$ For this reason, the ability to hemodynamically evaluate patients before and after surgical intervention is imperative, a perfect niche for ASL.

\section{ASL and Angiography}

Recently, several comparative studies have compared ASL against traditional angiography methods, including CTA, MRA, and catheter angiography. For example, a study by $\mathrm{Ha}$ et al. ${ }^{13}$ sought to correlate postoperative CBF measured by ASL with degree of revascularization as assessed by subtraction angiography in a cohort of 21 children with MMD. Using a normalized CBF metric, calculated by dividing the $\mathrm{CBF}$ of the MCA territory by the $\mathrm{CBF}$ of the cerebellum, these authors found normalized $\mathrm{CBF}$ values to increase significantly after the operation $(p=0.001)$. The amount of normalized CBF increase correlated with higher degrees of revascularization on catheter angiography ( $p=0.005$, Jonckheere-Terpstra test), providing evidence that ASL may be clinically useful in this setting. Another study by Yuan et al. ${ }^{44}$ compared territorial ASL (T-ASL) with CTA in 30 patients with MMD treated with STA-MCA bypass (Fig. 1). T-ASL selectively labels an individual artery, providing a vessel-specific rather than whole-brain perfusion map. In this study, the authors performed measurements of the ICAs, external carotid arteries (ECAs), and basilar artery, along with the perfusion territory of the bypass, denoted as the revascularization area. Patients in whom a revascularization area was identified had a more favorable outcome, confirming an increased blood supply from the ECA through the bypass. ${ }^{44}$ Quon et al. ${ }^{33}$ also showed ASL to be an efficacious noninvasive alternative to DSA in assessing postoperative cerebral perfusion changes after revascularization in MMD patients. In their cohort of 15 patients, ASL MRI showed significantly increased perfusion in the ipsilateral MCA territory $(\mathrm{p}=0.0059)$, which was correlated with 

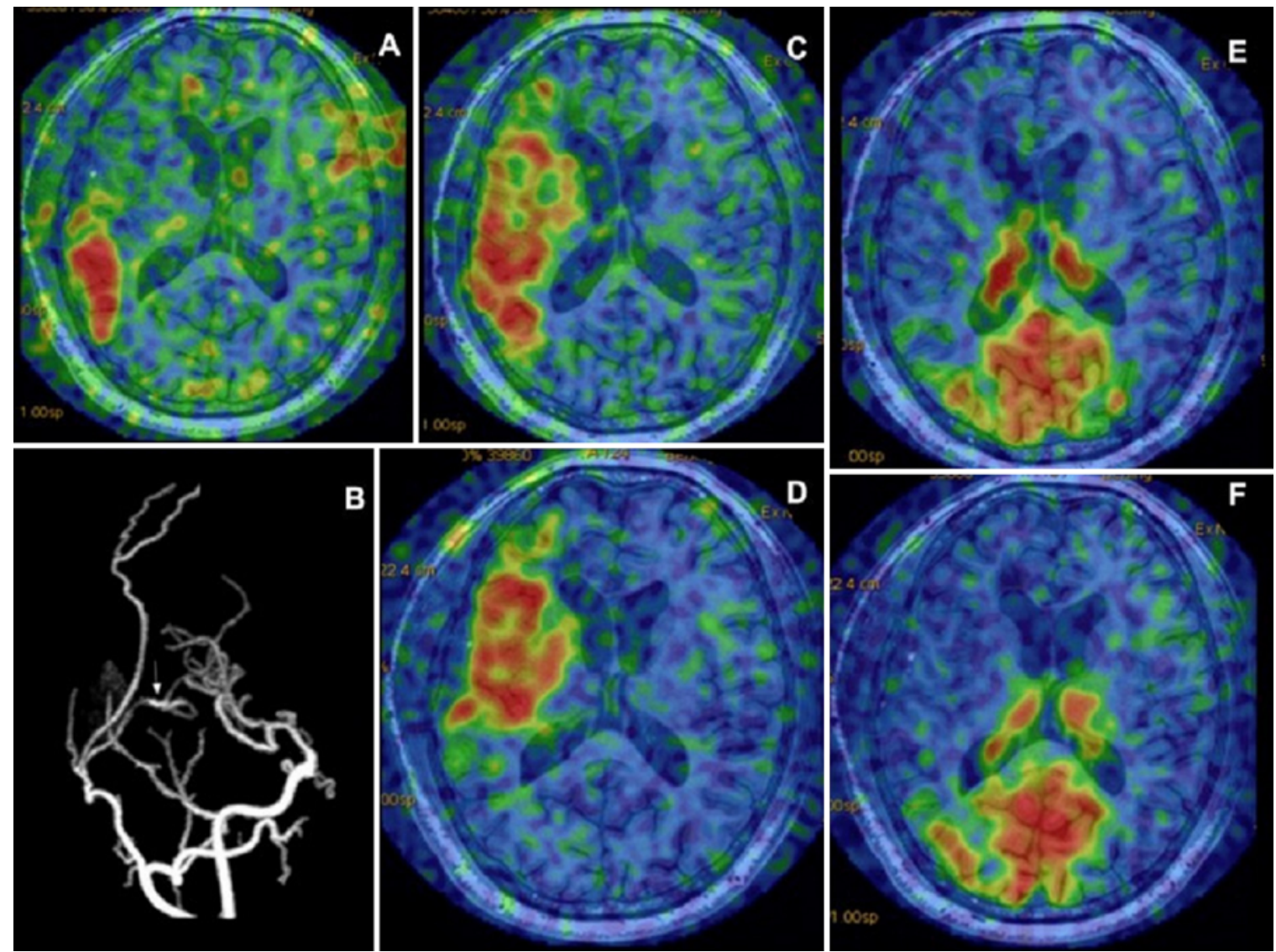

FIG. 1. Example of T-ASL in a patient who underwent STA-MCA revascularization for MMD. Postoperative T-ASL MR image (A) demonstrates a revascularization area (red) following bypass surgery. CTA (B) provides confirmation of the STA-MCA bypass (arrow) and patency. Comparison between preoperative (C) and postoperative (D) T-ASL images of the right ICA perfusion distribution. There is a marked reduction in the right ICA territory postoperatively given the reduced burden of perfusion as a result of the bypass surgery. Basilar artery perfusion remains unchanged when comparing preoperative $(E)$ and postoperative $(F)$ T-ASL images. Reprinted from World Neurosurgery, 122, Yuan J, Qu J, Zhang D, Liu X, Li J, Wu C, Gao P, Cerebral perfusion territory changes after direct revascularization surgery in moyamoya disease: a territory arterial spin labeling study, pp e1128-e1136, 2019, with permission from Elsevier. http://www.sciencedirect.com/journal/world-neurosurgery.

regression of collaterals seen prior to revascularization. In a study including 145 patients, Lee et al. ${ }^{24}$ published findings in agreement with those in other studies with respect to significant increases in $\mathrm{CBF}$ after revascularization ( $\mathrm{p}$ $<0.001$ in all CBF metrics analyzed). ASL grading of collaterals and anastomosis patency was well correlated with DSA findings, with a weighted kappa value of 0.77 (95\% CI 0.73-0.81) for MCA territory CBF. Also using kappa statistics to correlate ASL with DSA, Zaharchuk et al. ${ }^{46}$ identified a moderate to strong kappa value of $0.58(95 \%$ CI 0.52-0.64). The findings of these studies show that ASL provides a noninvasive method that is an efficacious alternative to traditional angiographic modalities.

\section{ASL and MRI}

Other studies have compared ASL to perfusion MRI. For example, Zhang et al. ${ }^{48}$ retrospectively explored the correlation between multi-inversion time ASL (mTI-ASL) and DSC-PWI in 24 patients with MMD. As in the study by Ha et al., ${ }^{13}$ relative perfusion values were attained by dividing by the cerebellum value, for both ASL- and
DSC-acquired images. Ultimately, their findings showed that mTI-ASL is a viable contrast-free alternative when assessing MMD preoperatively, with a strong correlation between relative $\mathrm{CBF}$ measured by ASL and that measured by DSC-PWI ( $r=0.839, \mathrm{p}<0.001)$, using Pearson correlation analysis in all ROIs analyzed (total of 90 lateral MCA territories and basal ganglia). ${ }^{48}$ Qiao et al ${ }^{31}$ also compared mTI-ASL and DSC MRI in quantifying CBF before and after revascularization in 41 MMD patients. The results showed that $\mathrm{CBF}$ measured by ASL and relative $\mathrm{CBF}$ measured by DSC in 824 ROIs were significantly correlated $(r=0.316, p<0.0001)$. In a study evaluating 10 MMD patients, Goetti et al. ${ }^{11}$ found ASL and DSC MRI to be strongly correlated in both qualitative perfusion assessment $(\rho=0.77, p<0.001)$ and quantitative relative CBF $(r=0.79, p<0.001)$. Good sensitivity, specificity, and accuracy were found as compared to DSC imaging (94\%, 93\%, 93\%, respectively). These findings are corroborated by Yun et al., ${ }^{45}$ who acquired ASL and DSC perfusion images in 54 patients with MMD. In their study, significant correlations were shown for the ICA- and MCA-based 
ROIs ( $r=0.877$ and 0.867 , respectively, $\mathrm{p}<0.0002$ for both). In addition, increasing the time to peak, a metric that measures the time from contrast injection to peak signal loss, to values greater than 25 seconds corresponded with increased correlation strength. ${ }^{45}$

Given the strong correlations found between ASL imaging and both angiography and perfusion MRI techniques, ASL has the potential to aid in clinical decision-making with respect to surgical planning such as identifying the STA and MCA prior to revascularization. ${ }^{39}$ However, further studies are needed to establish ASL as a leader in this domain.

\section{Tumor \\ Meningiomas}

ASL provides an opportunity for surgeons to preoperatively assess the grade and severity of meningiomas. MRI is helpful in categorizing the type of brain neoplasm but has not been utilized for grading. . $^{6,25}$ In a study of 54 patients with new or recurrent meningiomas, Qiao et al..$^{32}$ have demonstrated that ASL is a technique for differentiating WHO grade I from WHO grade II and III tumors based on tumor blood perfusion patterns. They showed that grade I tumors correspond to homogeneously hyperperfused signaling with a higher mean $\mathrm{CBF}$ relative to that in WHO grade II and III tumors, which display heterogeneously hyperperfused, isoperfused, or hypoperfused signaling with a lower mean CBF (Fig. 2). ${ }^{32}$ ASL has also been utilized to help reliably identify feeding arteries to meningiomas, an important element of preoperative surgical planning. In a study of 30 patients with suspected meningiomas, Lu et al. ${ }^{27}$ demonstrated that T-ASL was better able to assess nearby feeding branches $(\mathrm{kappa}=0.913)$ than MRA analysis (kappa $=0.653$ ). These investigators helped to differentiate whether the tumor in question was fed by the ECA or the ICA, thus aiding in assessment of the operative risk and surgical decision-making.

pCASL was used by Koizumi et al. ${ }^{18}$ to differentiate angiomatous from nonangiomatous meningiomas in a study of 25 patients. The investigators found a significant correlation between the microvascular density of the meningioma and the mean and maximum total tumor blood flow. The mean and maximum total tumor blood flow in angiomatous meningiomas were significantly higher than those in nonangiomatous meningiomas. ${ }^{18}$ As it stands, ASL serves as a novel clinical tool that provides additional information that can be integrated with various other conventional imaging techniques to aid in the assessment of and surgical planning for patients with meningiomas.

\section{Gliomas}

As in meningiomas, ASL can be utilized in the preoperative differentiation and staging of gliomas. In a study by Zeng et al., 3D pCASL was performed in 58 patients with a confirmed diagnosis of gliomas in order to understand blood flow relative to WHO grading. ${ }^{47}$ These authors found that relative CBF was significantly higher in high-grade gliomas than in low-grade gliomas. They also found that maximum $\mathrm{CBF}$ was a marker for both progression-free survival in gliomas and overall survival in glio- blastomas, with higher maximum relative CBFs leading to worse survival in patients with gliomas and better survival in those with glioblastomas. ${ }^{47}$ Even subtle hyperperfused tumor tissue seen on ASL MRI has been associated with progression in Ki-67-positive glioblastoma (Fig. 3). ${ }^{29}$

A recent meta-analysis has demonstrated that ASL is clinically useful in differentiating glioma grades on both PASL and pCASL. Falk Delgado et al. ${ }^{7}$ analyzed 15 studies with 505 patients and demonstrated that ASL helps to differentiate between high-grade and low-grade gliomas with a summary sensitivity of 0.89 (95\% CI $0.79-0.90)$ and specificity of 0.80 (95\% CI $0.72-0.89$ ). High-grade gliomas have a significant increase in all ASL perfusion values such that absolute tumor blood flow and maximum mean relative tumor blood flow aid in grading gliomas. ${ }^{19}$ These studies show that there is a difference in blood flow between high- and low-grade gliomas, but that specific grading of gliomas as classified by the WHO grading scale needs to be further investigated when specifically using ASL.

Given that the majority of studies for both meningiomas and gliomas relate to grading, diagnosis, and prognosis, there remains an increased need for studies assessing the utility of ASL and how it can affect decisions about tumor pathologies in ways other than determining grade.

\section{Other Disorders \\ Seizure}

Seizures are a pathological entity with mixed cerebral flow. Previous MRI, fMRI, PET, and SPECT studies have demonstrated significantly lower CBF during interictal states and increased CBF in ictal states..$^{35,38,43}$ The variety of CBF during these events offers an opportunity for periictal and ictal characterization via ASL analysis. Such characterization includes determining seizure focus, ascertaining abnormal peri-ictal perfusion patterns, and ruling out stroke in the setting of acute deficits. ${ }^{34}$

Studies of peri-ictal perfusion patterns have shown a postictal perfusion decrease of $71.4 \%(15 / 21)$ in patients studied with ASL. ${ }^{9}$ Of these patients, $80 \%$ showed areas of postictal hypoperfusion as demonstrated by ASL. These regions of hypoperfusion were partially or fully consistent with the location of presumed seizure onset. These findings are consistent with earlier work by Storti et al. ${ }^{37}$ who demonstrated that hypometabolism on FDG-PET analysis was correlated with hypoperfusion detected by ASL MRI. In a corollary to this principle of ictal hyperperfusion, ASL may help to rule out the similarly presenting stroke syndromes that demonstrate ictal hypoperfusion.

ASL has a role in establishing clinical seizure focus (Fig. 4). Kim et al. ${ }^{17}$ reported that $31 / 42$ patients with localizable clinical seizures had seizure foci that were positively identified by ASL MRI. Their work established the sensitivity and specificity of ASL in determining seizure foci at $74 \%$ (95\% CI 58\%-86\%) and 0\% (95\% CI 0\%$84 \%$ ), respectively. Despite clinical seizure foci that were not localizable in 2 patients, ASL imaging inappropriately identified seizure foci, leading to the low specificity in the study. Therefore, Kim and colleagues claimed that the overall accuracy of ASL in their study was $70 \%$. The au- 

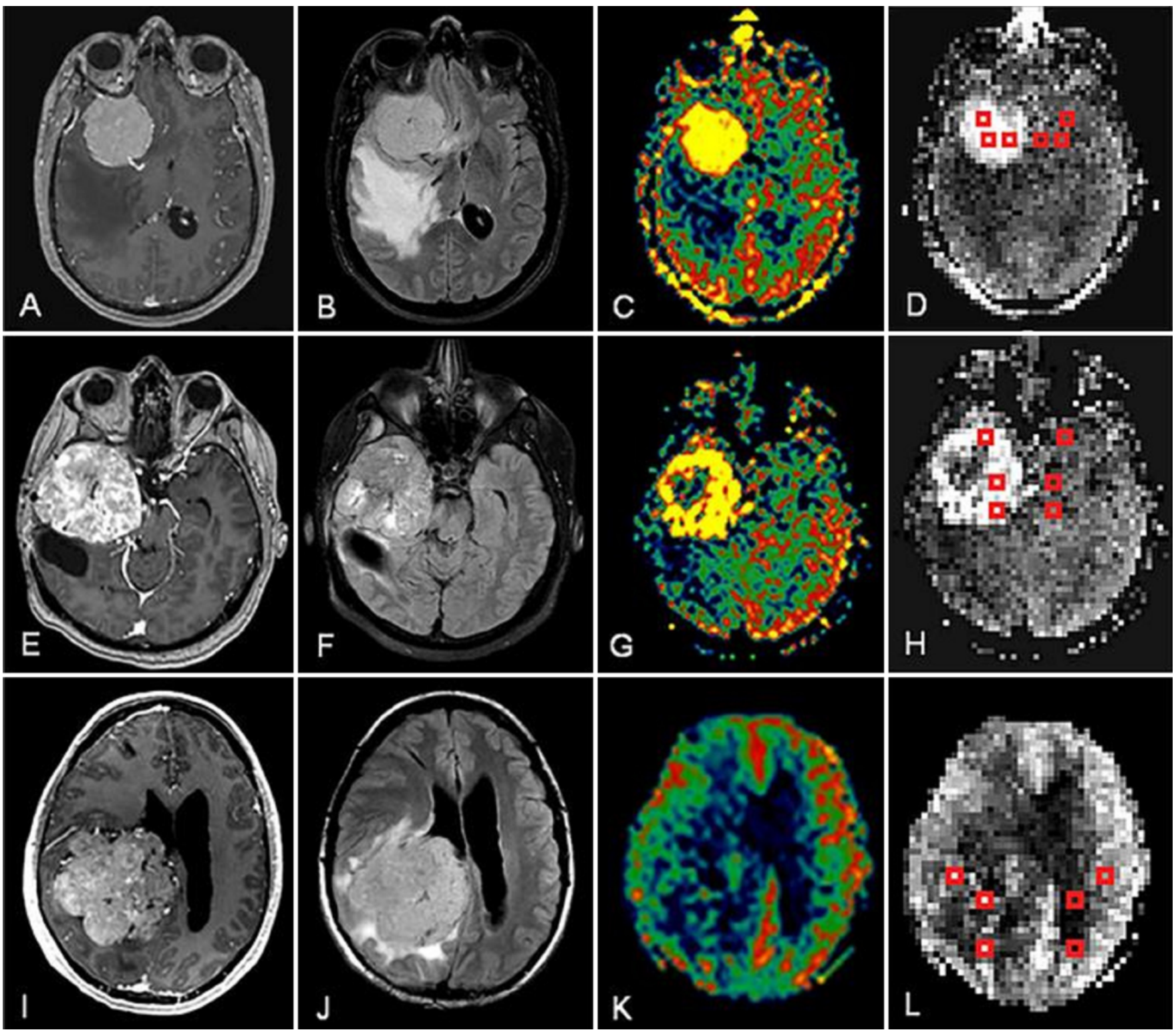

FIG. 2. Comparison between conventional MRI and ASL CBF perfusion maps in patients with meningioma. Postcontrast T1weighted images (A, E, and I) and T2-FLAIR sequences (B, F, and J) from 3 patients, respectively. ASL perfusion images from 3 patients with three distinct perfusion patterns: homogeneous hyperperfused tumor tissue (bright yellow, $\mathbf{C}$ ) versus heterogeneous hyperperfused tumor tissue (bright mixed yellow, G) versus iso- or hypoperfused tumor region (green/blue, K). Manually set ROls (red squares, $\mathrm{D}, \mathrm{H}$, and $\mathrm{L}$ ) that aid in the quantification of both tumor and contralateral brain regions. Reprinted from European Journal of Radiology, 97, Qiao XJ, Kim HG, Wang DJJ, Salamon N, Linetsky M, Sepahdari A, Ellingson BM, Pope WB, Application of arterial spin labeling perfusion MRI to differentiate benign from malignant intracranial meningiomas, pp 31-36, 2017, with permission from Elsevier. https://www.journals.elsevier.com/european-journal-of-radiology.

thors noted the failure of ASL to identify small hypoperfusion in the temporal lobe; however, they predict that other imaging and exam modalities in the clinical setting will compensate for this drawback of ASL perfusion imaging. ASL has also been correlated with electroencephalography (EEG) findings of seizure foci in $72 \%$ of patients. ${ }^{34}$ Therefore, ASL seems to be an emerging modality whose findings agree with data establishing increased perfusion and EEG spikes at epileptogenic foci.

In fact, the relationship between EEG and ASL per- fusion findings has been studied in pediatric populations. Pediatric patients with acute seizures and no abnormalities on structural MRI were studied with ASL and EEG. The study by Lee et al. ${ }^{23}$ showed concordance (kappa = 0.542 ) between ASL findings and clinical seizure focus. Therefore, ASL may have a role in evaluating epilepsy in the setting of normal structural MRI.

\section{Migraines}

ASL has clinical utility in assessing migraines (Fig. 5). 

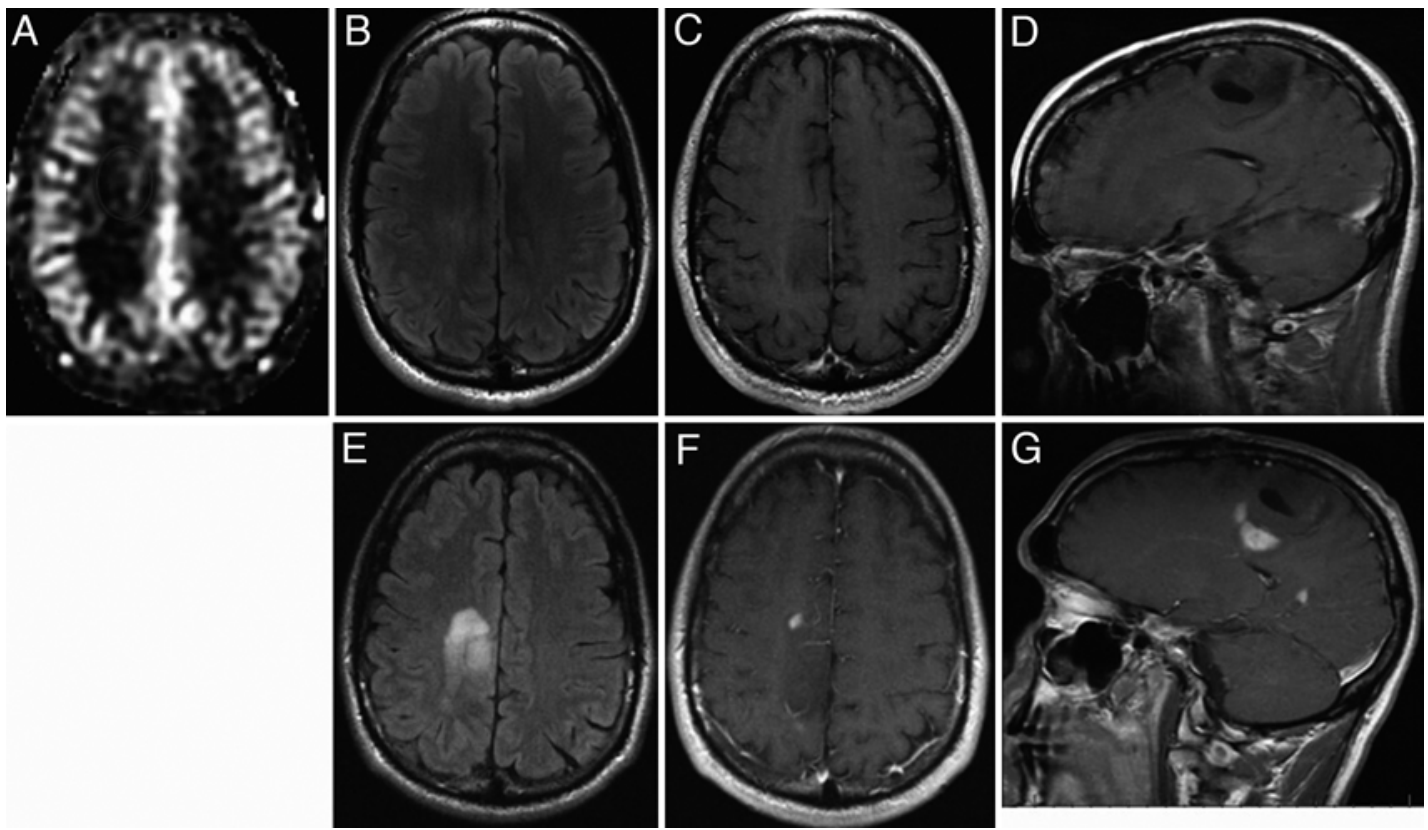

FIG. 3. Images obtained in a patient with a history of a WHO grade II glioma that had been treated 9 years earlier and presenting with seizures. ASL imaging (A) depicts a subtle hyperperfusion change of $34 \mathrm{ml} / 100 \mathrm{~g} / \mathrm{min}$. A relative CBF (rCBF) ratio elevation was apparent at 1.9. FLAIR (B) and contrast-enhanced T1-weighted (C and D) images are unrevealing. Repeat imaging 2 months later demonstrated tumor progression on FLAIR $(E)$ and contrast-enhanced T1-weighted (F and $\mathbf{G}$ ) images, although no ASL imaging was obtained at this time. Biopsy was remarkable for a hypercellular infiltrating WHO grade IV glioblastoma positive for Ki-67 labeling, IDH1 mutation, and p53 immunoreactivity, suggesting that subtle CBF changes in ASL may represent evidence of tumor progression. Nyberg E, Honce J, Kleinschmidt-DeMasters BK, Shukri B, Kreidler S, Nagae L, Neuroradiology Journal (Vol 29, Issue 5), pp 377-383, @2016 by the authors. Reprinted by permission of SAGE Publications, Ltd.

In a group of 49 pediatric and adolescent patients, $11(22 \%)$ demonstrated perfusion abnormalities on ASL, with the occipital lobe being the most commonly involved (73\%). ${ }^{40}$ Of these 11 patients, 10 had hyperperfusion abnormalities. Moreover, patients with these abnormalities showed a significant difference in hospitalization, confusion, motor disability, and aura symptoms compared to those in patients without ASL-established perfusion abnormalities.

This finding is somewhat consistent with other works, demonstrating that cerebral hyperperfusion (as established by ASL) may be correlated to the pathophysiology of migraine presentation. ${ }^{4}$ However, other studies have correlated migraines with mixed hyperperfusion/hypoperfusion episodes ${ }^{42}$ and others still point primarily to hypoperfusion on ASL during episodes of migraine with aura. ${ }^{22}$ Regardless, these studies seem to agree that their exists perfusion abnormality on ASL in patients who have migraine with aura. In patients who have migraine without aura, however, there may not be any perfusion abnormality as assessed by ASL. ${ }^{10}$

One case series of pediatric patients has demonstrated that among ASL, susceptibility weighted imaging, MRA, and diffusion weighted imaging, ASL is the most sensitive in detecting hemiplegic migraine. ${ }^{5}$ Therefore, ASL may have utility in differentiating between hemiplegic migraine and stroke. As regards perfusion abnormality, the same study used ASL to demonstrate that initial hypoperfusion in migraine was followed by rebound hyperperfusion. ${ }^{5}$ Thus, the discrepancy in whether migraine is correlated with hypoperfusion or hyperperfusion may instead be explained by the chronicity of perfusion changes in migraine presentation, as demonstrated by ASL imaging.

\section{Recent Advances in ASL Imaging}

Given the potential advantages of ASL over other perfusion-based techniques, research is ongoing with efforts to increase image quality and ease of use. Mora Álvarez et al. ${ }^{28}$ obtained high-resolution images using a CASL technique by combining a separate neck coil to another coil used during RF excitation during a 6-minute scan time on 4.7-T MRI. The higher resolution was demonstrated in comparison to standard $2 \mathrm{D}$ and qualitative perfusion images in the same subjects; the method also showed superior perfusion visibility in small deep-brain structures. This could have clinical relevance in detecting perfusion deficits in smaller areas of the brain, which are more likely to go undetected in lower-resolution techniques..$^{28,31,46}$

Another problem in ASL results from difficulties in predicting the optimum PLD; while multiphase PLD may respond to varying arterial flow rates, it is prone to lower SNR, making single-phase PLD the preferred method in clinical practice. However, this method can lead to inaccuracies in predicting peak flow rates, making image capture at the most appropriate time to maximize image quality difficult and necessitating imaging at multiple PLD patterns. ${ }^{30}$ Oshita et al. developed a method of correcting PLD for an individual patient by using 4D MRA times modified by an algorithm based on patient data to predict flow times for individual vascular territories..$^{30}$ This tech- 


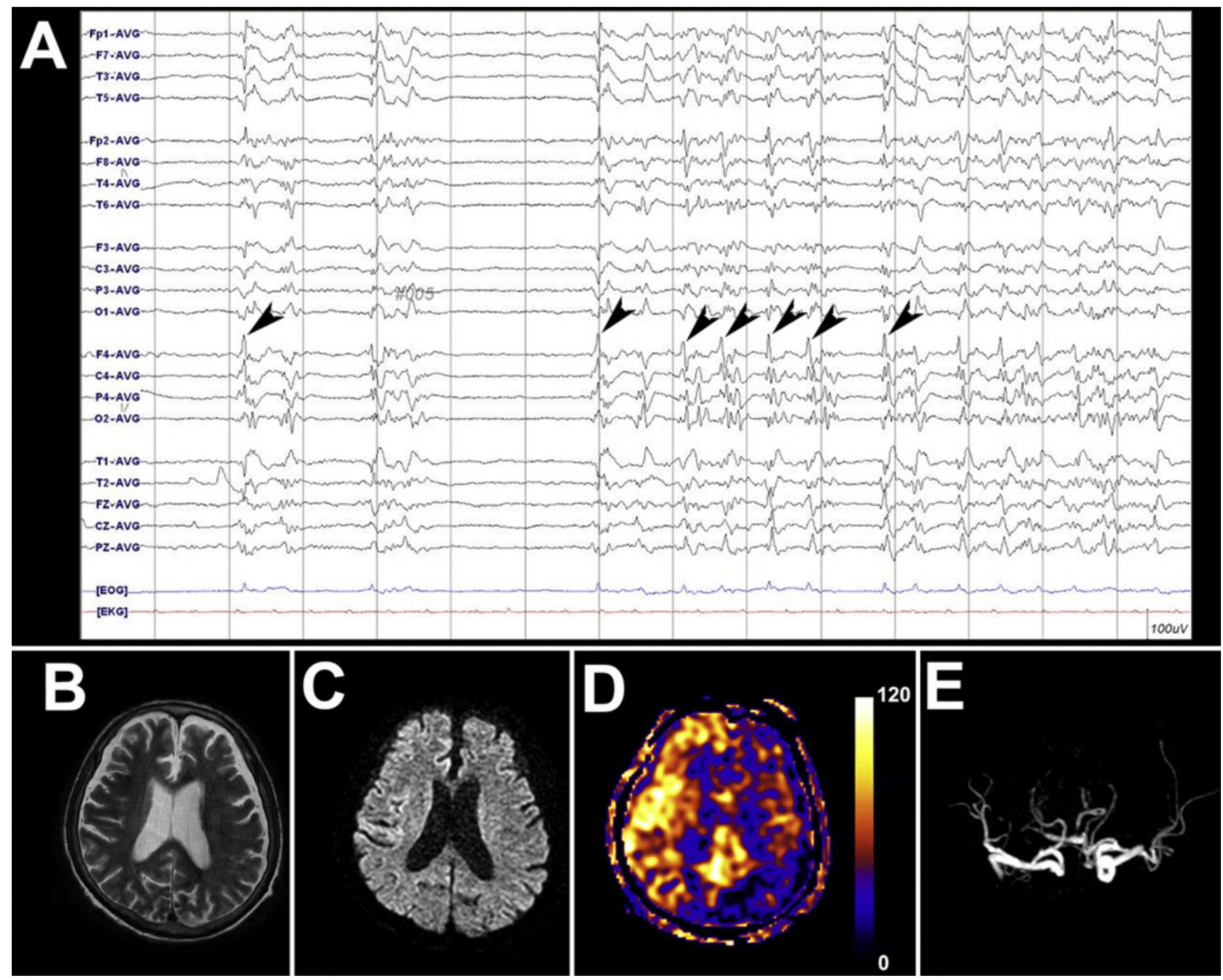

FIG. 4. Data on a patient presenting with nonconvulsive status epilepticus. Electroencephalography recording (A) depicting periodic epileptiform discharge in the right frontocentral area (arrowheads). Axial T2-weighted (B) and diffusion weighted (C) images show no abnormal lesions. ASL perfusion image (D) demonstrates hyperperfusion in the right hemisphere, measured in units of $\mathrm{ml} / 100 \mathrm{~g} / \mathrm{min}$ on the right axis. MR angiogram (E) demonstrates full patency of intracranial arteries without occlusion. Reprinted from European Journal of Radiology, Vol 85, Kim BS, Lee ST, Yun TJ, Lee SK, Paeng JC, Jun J, Kang KM, Choi SH, Kim J, Sohn $\mathrm{CH}$, Capability of arterial spin labeling MR imaging in localizing seizure focus in clinical seizure activity, pp 1295-1303, @2016, with permission from Elsevier. https://www.journals.elsevier.com/european-journal-of-radiology.

nique provides the advantage of reducing imaging time, as reliance on multiple PLD patterns is decreased, and can theoretically mitigate individual patient variations such as degree of arterial stenosis, underlying diseases, age, and sex; however, the technique still requires validation in a larger cohort of patients. ${ }^{30}$

ASL data are typically produced at a voxel size of 3-5 $\mathrm{mm}$, meaning that it has difficulty capturing small enough regions to limit the data to a single tissue type; this leads to a bias known as the partial volume (PV) effect, which is particularly problematic in perfusion imaging because of the significantly different perfusion properties between the gray and white matter in voxels containing mixed tissue. ${ }^{49}$ Liu et al. attempted to correct the PV effect while accounting for mixed tissue by creating a structure-based expectation maximization (sEM) to estimate perfusion of the mixed elements of voxels containing different tissue types. ${ }^{26}$ Compared to the linear regression method, the sEM model preserved more detail from tissue interfaces and brain lesions, increasing measurements of CBF. This resulted in better sensitivity in the detection of small lesions and allowed for a reduced scan time given the need for fewer label/control pairs.

\section{Conclusions}

As ASL imaging continues to be refined, its application has expanded into the realm of neurovascular disorders, tumors, and other nervous system ailments such as seizures and migraines. As evidenced by the studies described, 

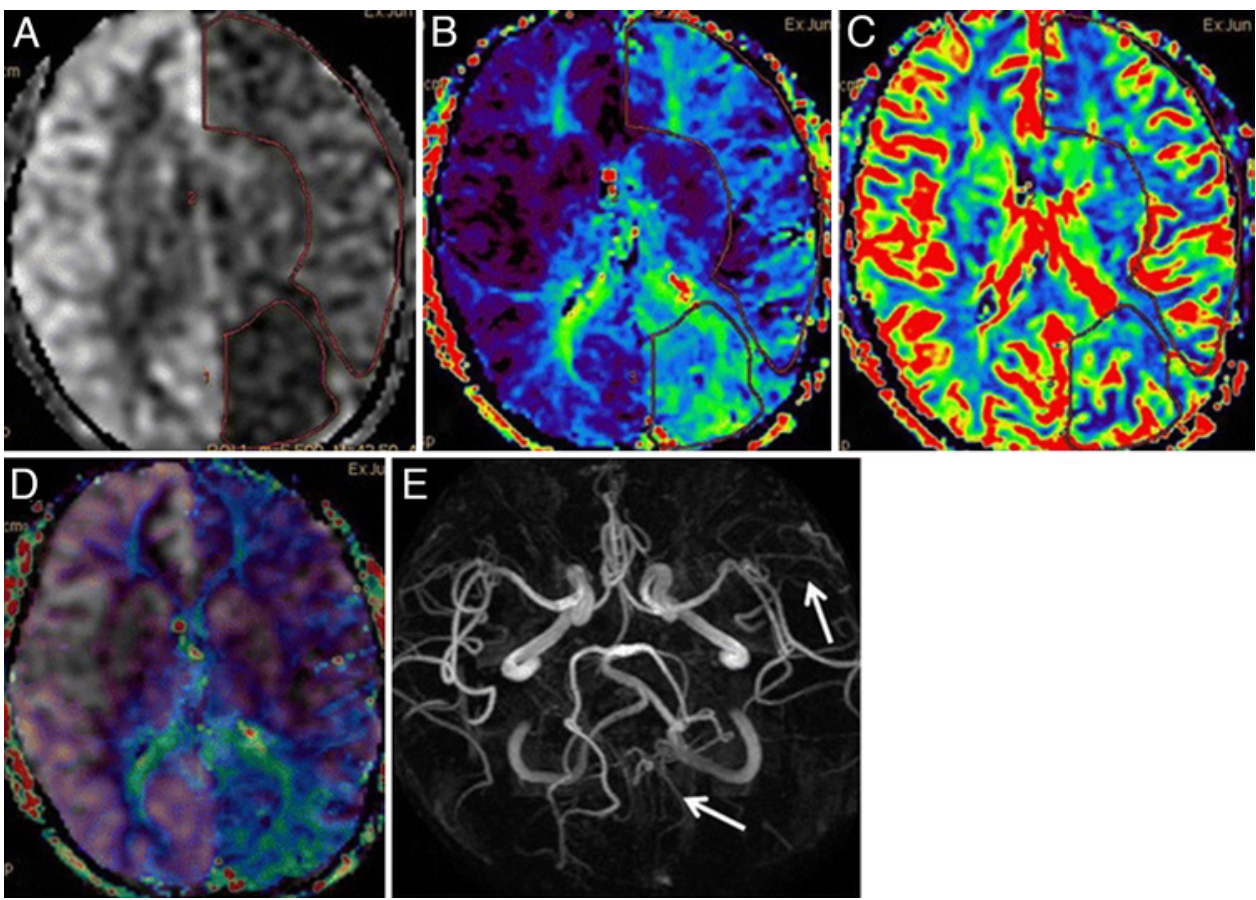

FIG. 5. Example of ASL in a pediatric patient having a hemiplegic migraine. pCASL perfusion map (A) depicts areas of hypoperfusion predominating the left posterior cerebral hemisphere. A mean CBF of $17.5 \mathrm{ml} / 100 \mathrm{~g} / \mathrm{min}$ over the parietooccipital area was found as compared to $27.4 \mathrm{ml} / 100 \mathrm{~g} / \mathrm{min}$ in the frontoparietal area. DSC-PWI color-coded parametric perfusion maps (B and C). $\mathrm{ROI}$ analysis indicated a time to max (Tmax) delay of 4.5 seconds in the parietooccipital area, which is higher than that in the frontoparietal area with a Tmax delay of 3.3 seconds (B). ROI analysis over the left parietooccipital area showed a mean regional CBF (rCBF) of $44.15 \mathrm{ml} / 100 \mathrm{~g} / \mathrm{min}$ compared to $47.18 \mathrm{ml} / 100 \mathrm{~g} / \mathrm{min}$ in the left frontoparietal area (C). Fusion of the initial ASL and DSC-PWI perfusion maps (D) shows hypoperfusion in the ASL perfusion map to correspond with the areas of hypoperfusion as seen on the Tmax and rCBF maps of DSC-PWI. An MRA study (E) showing vasoconstriction of peripheral branches (white arrows) of the left MCA and left posterior cerebral artery. Reprinted by permission from Springer Nature Customer Service Centre GmbH: Springer, European Journal of Pediatrics, A case report of sporadic hemiplegic migraine associated cerebral hypoperfusion: comparison of arterial spin labeling and dynamic susceptibility contrast perfusion MR imaging, Kim S, Kang M, Choi S, @2016. https:// link.springer.com/journal/431.

brain pathology is characterized by unique neurovascular physiology. These changes in CBF are detectable by several modalities, and ASL is the safest and least invasive of those available. Especially in the realm of MMD, which predominantly affects children, it is beneficial to use the least invasive approach without the use of contrast. In addition, ASL lends itself to the postoperative assessment of patients who have undergone revascularization, allowing neurosurgeons to test the efficacy of their bypass. As it stands, ASL imaging continues to be used as an adjuvant rather than a standalone imaging modality and has a long way to go before replacing conventional neuroimaging techniques.

\section{References}

1. Alsop DC, Detre JA, Golay X, Günther M, Hendrikse J, Hernandez-Garcia L, et al: Recommended implementation of arterial spin-labeled perfusion MRI for clinical applications: a consensus of the ISMRM perfusion study group and the European consortium for ASL in dementia. Magn Reson Med 73:102-116, 2015

2. Borogovac A, Asllani I: Arterial spin labeling (ASL) fMRI: advantages, theoretical constrains, and experimental challenges in neurosciences. Int J Biomed Imaging 2012:818456, 2012
3. Chen JJ, Jann K, Wang DJJ: Characterizing resting-state brain function using arterial spin labeling. Brain Connect 5:527-542, 2015

4. Chen Z, Chen X, Liu M, Liu M, Ma L, Yu S: Evaluation of gray matter perfusion in episodic migraine using voxel-wise comparison of 3D pseudo-continuous arterial spin labeling. J Headache Pain 19:36, 2018

5. Cobb-Pitstick KM, Munjal N, Safier R, Cummings DD, Zuccoli G: Time course of cerebral perfusion changes in children with migraine with aura mimicking stroke. AJNR Am J Neuroradiol 39:1751-1755, 2018

6. Detre JA, Leigh JS, Williams DS, Koretsky AP: Perfusion imaging. Magn Reson Med 23:37-45, 1992

7. Falk Delgado A, De Luca F, van Westen D, Falk Delgado A: Arterial spin labeling MR imaging for differentiation between high- and low-grade glioma-a meta-analysis. Neuro Oncol 20:1450-1461, 2018

8. Fujimura M, Bang OY, Kim JS: Moyamoya disease. Front Neurol Neurosci 40:204-220, 2016

9. Gaxiola-Valdez I, Singh S, Perera T, Sandy S, Li E, Federico P: Seizure onset zone localization using postictal hypoperfusion detected by arterial spin labelling MRI. Brain 140:2895-2911, 2017

10. Gil-Gouveia R, Pinto J, Figueiredo P, Vilela PF, Martins IP: An arterial spin labeling MRI perfusion study of migraine without aura attacks. Front Neurol 8:280, 2017

11. Goetti R, O'Gorman R, Khan N, Kellenberger CJ, Scheer 
I: Arterial spin labelling MRI for assessment of cerebral perfusion in children with moyamoya disease: comparison with dynamic susceptibility contrast MRI. Neuroradiology 55:639-647, 2013

12. Grade M, Hernandez Tamames JA, Pizzini FB, Achten E, Golay X, Smits M: A neuroradiologist's guide to arterial spin labeling MRI in clinical practice. Neuroradiology 57:11811202,2015

13. Ha JY, Choi YH, Lee S, Cho YJ, Cheon JE, Kim IO, et al: Arterial spin labeling MRI for quantitative assessment of cerebral perfusion before and after cerebral revascularization in children with moyamoya disease. Korean J Radiol 20:985-996, 2019

14. Hendrikse J, Petersen ET, Golay X: Vascular disorders: insights from arterial spin labeling. Neuroimaging Clin N Am 22:259-269, x-xi, 2012

15. Jezzard P, Chappell MA, Okell TW: Arterial spin labeling for the measurement of cerebral perfusion and angiography. J Cereb Blood Flow Metab 38:603-626, 2018

16. Kawahara Y, Nakada M, Hayashi Y, Kai Y, Hayashi Y, Uchiyama N, et al: Prediction of high-grade meningioma by preoperative MRI assessment. J Neurooncol 108:147-152, 2012

17. Kim BS, Lee ST, Yun TJ, Lee SK, Paeng JC, Jun J, et al: Capability of arterial spin labeling MR imaging in localizing seizure focus in clinical seizure activity. Eur J Radiol 85:1295-1303, 2016

18. Koizumi S, Sakai N, Kawaji H, Takehara Y, Yamashita S, Sakahara H, et al: Pseudo-continuous arterial spin labeling reflects vascular density and differentiates angiomatous meningiomas from non-angiomatous meningiomas. J Neurooncol 121:549-556, 2015

19. Kong L, Chen H, Yang Y, Chen L: A meta-analysis of arterial spin labelling perfusion values for the prediction of glioma grade. Clin Radiol 72:255-261, 2017

20. Koretsky AP: Early development of arterial spin labeling to measure regional brain blood flow by MRI. Neuroimage 62:602-607, 2012

21. Krishnamurthy R, Wang DJJ, Cervantes B, McAllister A, Nelson E, Karampinos DC, et al: Recent advances in pediatric brain, spine, and neuromuscular magnetic resonance imaging techniques. Pediatr Neurol 96:7-23, 2019

22. Law-Ye B, Fargeot G, Leclercq D: Arterial spin labeling hypoperfusion in migraine aura. Headache 57:935-936, 2017

23. Lee SM, Kwon S, Lee YJ: Diagnostic usefulness of arterial spin labeling in MR negative children with new onset seizures. Seizure 65:151-158, 2019

24. Lee S, Yun TJ, Yoo RE, Yoon BW, Kang KM, Choi SH, et al: Monitoring cerebral perfusion changes after revascularization in patients with moyamoya disease by using arterial spin-labeling MR imaging. Radiology 288:565-572, 2018

25. Lin BJ, Chou KN, Kao HW, Lin C, Tsai WC, Feng SW, et al: Correlation between magnetic resonance imaging grading and pathological grading in meningioma. J Neurosurg 121:1201-1208, 2014

26. Liu Y, Wang Z, Liang R, Liang Z, Lu H: Partial volume correction for arterial spin labeling using the inherent perfusion information of multiple measurements. Biomed Eng Online 18:12, 2019

27. Lu Y, Luan S, Liu L, Xiong J, Wen J, Qu J, et al: Evaluation of the applicability of territorial arterial spin labeling in meningiomas for presurgical assessments compared with 3-dimensional time-of-flight magnetic resonance angiography. Eur Radiol 27:4072-4081, 2017

28. Mora Álvarez MG, Stobbe RW, Beaulieu C: High resolution continuous arterial spin labeling of human cerebral perfusion using a separate neck tagging RF coil. PLoS One 14:e0215998, 2019

29. Nyberg E, Honce J, Kleinschmidt-DeMasters BK, Shukri B, Kreidler S, Nagae L: Arterial spin labeling: Pathologically proven superiority over conventional MRI for detection of high-grade glioma progression after treatment. Neuroradiol J 29:377-383, 2016

30. Oshita T, Yabuuchi H, Osame M: Optimization of post-label delay in single-phase arterial spin labeling (ASL) using multi-phase ASL in four-dimensional magnetic resonance angiography. Neuroradiol J 32:173-178, 2019

31. Qiao PG, Han C, Zuo ZW, Wang YT, Pfeuffer J, Duan L, et al: Clinical assessment of cerebral hemodynamics in moyamoya disease via multiple inversion time arterial spin labeling and dynamic susceptibility contrast-magnetic resonance imaging: a comparative study. J Neuroradiol 44:273-280, 2017

32. Qiao XJ, Kim HG, Wang DJJ, Salamon N, Linetsky M, Sepahdari A, et al: Application of arterial spin labeling perfusion MRI to differentiate benign from malignant intracranial meningiomas. Eur J Radiol 97:31-36, 2017

33. Quon JL, Kim LH, Lober RM, Maleki M, Steinberg GK, Yeom KW: Arterial spin-labeling cerebral perfusion changes after revascularization surgery in pediatric moyamoya disease and syndrome. J Neurosurg Pediatr 23:486-492, 2019

34. Schertz J, Benzakoun M, Pyatigorskaya N, Belkacem S, Sahli-Amor M, Navarro V, et al: Specificities of arterial spin labeling (ASL) abnormalities in acute seizure. J Neuroradiol [epub ahead of print], 2018

35. Schwartz TH: Neurovascular coupling and epilepsy: hemodynamic markers for localizing and predicting seizure onset. Epilepsy Curr 7:91-94, 2007

36. Soldozy S, Costello JS, Norat P, Sokolowski JD, Soldozy K, Park MS, et al: Extracranial-intracranial bypass approach to cerebral revascularization: a historical perspective. Neurosurg Focus 46(2):E2, 2019

37. Storti SF, Boscolo Galazzo I, Del Felice A, Pizzini FB, Arcaro C, Formaggio E, et al: Combining ESI, ASL and PET for quantitative assessment of drug-resistant focal epilepsy. Neuroimage 102:49-59, 2014

38. Telischak NA, Detre JA, Zaharchuk G: Arterial spin labeling MRI: clinical applications in the brain. J Magn Reson Imaging 41:1165-1180, 2015

39. Uchino H, Ito M, Fujima N, Kazumata K, Yamazaki K, Nakayama N, et al: A novel application of four-dimensional magnetic resonance angiography using an arterial spin labeling technique for noninvasive diagnosis of Moyamoya disease. Clin Neurol Neurosurg 137:105-111, 2015

40. Uetani H, Kitajima M, Sugahara T, Kikuchi H, Muto Y, Hirahara T, et al: Perfusion abnormality on three-dimensional arterial spin labeling with a 3T MR system in pediatric and adolescent patients with migraine. J Neurol Sci 395:41-46, 2018

41. Williams DS, Detre JA, Leigh JS, Koretsky AP: Magnetic resonance imaging of perfusion using spin inversion of arterial water. Proc Natl Acad Sci U S A 89:212-216, 1992

42. Wolf ME, Okazaki S, Eisele P, Rossmanith C, Gregori J, Griebe M, et al: Arterial spin labeling cerebral perfusion magnetic resonance imaging in migraine aura: an observational study. J Stroke Cerebrovasc Dis 27:1262-1266, 2018

43. Wu RH, Bruening R, Noachtar S, Arnold S, Berchtenbreiter $\mathrm{C}$, Bartenstein P, et al: MR measurement of regional relative cerebral blood volume in epilepsy. J Magn Reson Imaging 9:435-440, 1999

44. Yuan J, Qu J, Zhang D, Liu X, Li J, Wu C, et al: Cerebral perfusion territory changes after direct revascularization surgery in moyamoya disease: a territory arterial spin labeling study. World Neurosurg 122:e1128-e1136, 2019

45. Yun TJ, Sohn CH, Han MH, Kang HS, Kim JE, Yoon BW, et al: Effect of delayed transit time on arterial spin labeling: correlation with dynamic susceptibility contrast perfusion magnetic resonance in moyamoya disease. Invest Radiol 48:795-802, 2013 
46. Zaharchuk G, Do HM, Marks MP, Rosenberg J, Moseley ME, Steinberg GK: Arterial spin-labeling MRI can identify the presence and intensity of collateral perfusion in patients with moyamoya disease. Stroke 42:2485-2491, 2011

47. Zeng Q, Jiang B, Shi F, Ling C, Dong F, Zhang J: 3D pseudocontinuous arterial spin-labeling MR imaging in the preoperative evaluation of gliomas. AJNR Am J Neuroradiol 38:1876-1883, 2017

48. Zhang J, Xia C, Liu Y, Qian W, Peng W, Liu K, et al: Comparative study of MR mTI-ASL and DSC-PWI in evaluating cerebral hemodynamics of patients with moyamoya disease. Medicine (Baltimore) 97:e12768, 2018

49. Zhao MY, Mezue M, Segerdahl AR, Okell TW, Tracey I, Xiao Y, et al: A systematic study of the sensitivity of partial volume correction methods for the quantification of perfusion from pseudo-continuous arterial spin labeling MRI. Neuroimage 162:384-397, 2017

\section{Disclosures}

The authors report no conflict of interest concerning the materials or methods used in this study or the findings specified in this paper.

\section{Author Contributions}

Conception and design: Soldozy. Acquisition of data: Soldozy, Galindo, Snyder, Ali. Drafting the article: Soldozy, Galindo, Snyder, Ali. Critically revising the article: Soldozy, Norat, Yağmurlu. Reviewed submitted version of manuscript: all authors. Approved the final version of the manuscript on behalf of all authors: Kalani. Administrative/technical/material support: Kalani, Sokolowski, Sharifi, Tvrdik, Park. Study supervision: Kalani, Tvrdik, Park.

\section{Correspondence}

M. Yashar S. Kalani: University of Virginia Health System, Charlottesville, VA. kalani@virginia.edu. 